\title{
Yarn End Retrieving from Bobbins in Axisymmetric Accelerated Flow
}

Part 3: Effect of Canal Shapes along Chases

\author{
By Tsuneo Hirai*†, Masashi Inoue*0, Tsutao Katayama**†, Naomichi Heya ${ }^{* * * \dagger}$ and Sadasuke Fukai ${ }^{* * * * \dagger}$ \\ *Faculty of Engineering, Doshisha University, Kyoto \\ **Osaka Prefectural Industrial Research Institute, Osaka \\ ***Faculty of Engineering, Fukui Univercity, Fukui \\ ****Faculty of Engineering, Osaka City University, Osaka \\ $\dagger$ Member, TMSJ \\ ${ }^{\circ}$ Present Address; Toyobo Co., Ltd., Mie
}

Based on Journal of the Textile Machinery Society of Japan, Transactions, Vol. 32, No. 12, T96-103 (1979)

\begin{abstract}
The effect of shapes of air canals along the chase is investigated to find an effective method for yarn end retrieving from the point of energy-saving, by using cylindrical, conical or intermediate air guides as hoods. The flow patterns in the axisymmetrical canals between the bobbin and the hood attached to it can be understood by the conclusion of the previous paper thet the retrieving effect is dependent on the vertical component of the accelerated flow.

Experimental results here obtained show that, when the ratio of the minimum cylindrical inner diameter to the diameter at the skirt entrance is 0.79 , the flow pattern would become very effective to retrieve yarn ends from the conical surface of the bobbin.
\end{abstract}

\section{Introduction}

In previous papers ${ }^{[1,2]}$, it was considered how the entrance shapes of a hood has influence upon the efficiency of the yarn end retrieving when the air flow through an axisymmetric narrow gap between a bobbin and a hood retrieves and carries away the yarn end. The results showed that, for yarn end retrieving, the existence of flow acceleration, especially variation of acceleration was effective rather than the flow speed itself and there were some effective entrance guides for air inlet. Also, it was pointed out that the study for the canal shape should be carried out to prevent the yarn at the top edge of the chase from sloughing out of the bobbin.

In this paper, assuming that the broken end of a yarn exists at the chase of a bobbin similarly to the previous experiments, the ability of yarn end retrieving from a bobbin is tested in cases of various shapes of the canal between the bobbin and its hood. The flow pattern in the canal along the chase is guessed by the pressure distributions measured on the inner surface of the hood and on the bobbin using a model bobbin. Consequently, effective conditions for yarn end retrieving by air suction, especially effective exit shapes at the top of the chase, are considered.

\section{Experimental Apparatus and Procedure}

The experimental apparatus used in the present study is the same as that used in the previous paper ${ }^{[1]}$. All hoods travel downward to overlap a bobbin until a gap clearance is $2.0 \mathrm{~mm}$. After they stop there for $0.2 \mathrm{sec}$, they travel upward again. Shapes and dimensions of hoods used are shown in Fig. 1. Hood A', B' and $C^{\prime}$ have a taper part of angle $12^{\circ}$. Hood D' is cylindrical. The ratio of the inner diameter of the hood at the top edge of a chase to the inner diameter of the hood entrance is 0.54 for Hood A', 0.61 for Hood B', 0.79 for Hood C' and 1.00 for Hood D'.

It was very difficult to suck the broken end of the yarn in the previous paper when 60 s of polyester yarn was used. In this experiments the relationship between the yarn end retrieving efficiency and the air flow rate is studied at the ambient temperature of $18 \pm 2^{\circ} \mathrm{C}$ and the relative humidity of $65 \pm 10 \%$. For each test, the yarn end was cut with scissors and pressed softly on the chase.

In order to consider the effective canal shape for yarn end retrieving the wall pressure distributions of the air flow on the inner surface of a hood and on a bobbin are measured. The experimental apparatus and a model bobbin used are 

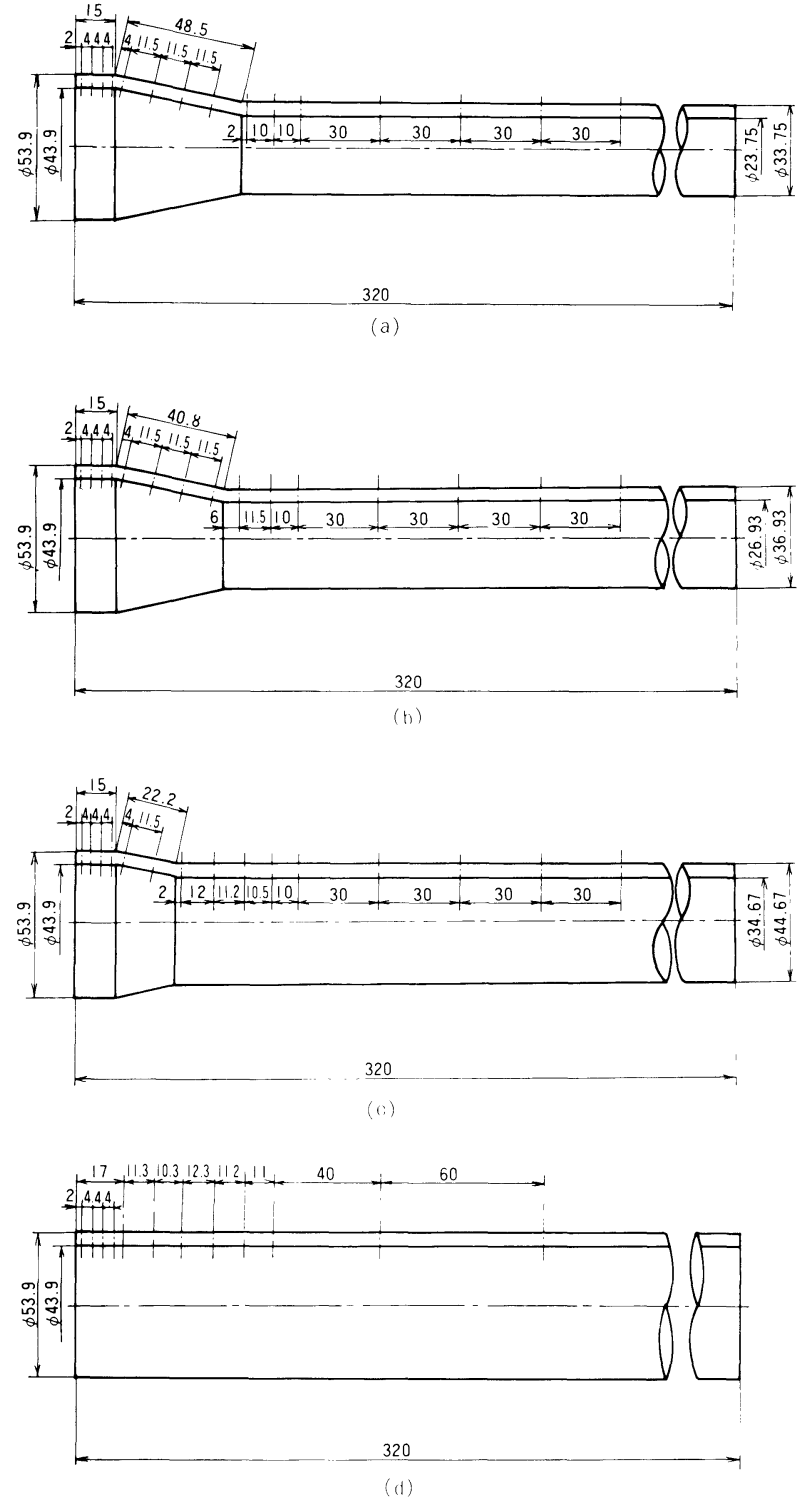

Fig. 1 Shapes and dimensions of hoods used for experiments (a) Hood A' (b) Hood B' (c) Hood C' (d) Hood D'

the same as those in the previous paper ${ }^{[2]}$. The gap clearance between the inner surface of the hood and the chase surface of the model bobbin are 1.0, 2.0 and $3.0 \mathrm{~mm}$. The pressure measuring taps are shown in Fig. 1 on hoods. Those for the model bobbin are shown in the previous paper ${ }^{[2]}$.

\section{Results and Discussion}

Fig. 2 shows the relationship between the yarn end retrieving efficiency and the air flow rate when yarn ends are placed at the top, at the middle and at the bottom end of the bobbin chase. The efficiency of the yarn end retrieving is defined as

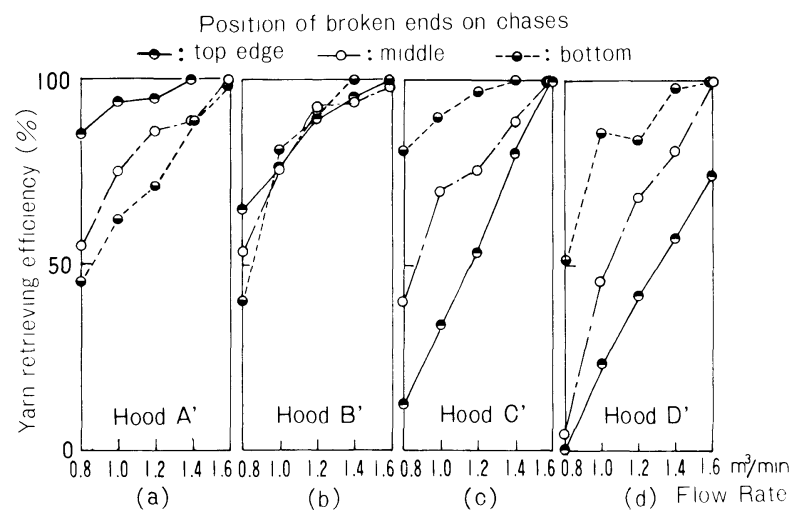

Fig. 2 Relationship between the retrieving efficiency and the flow rate

the number of success per one hundred trials. The gap clearance between the hood and the bobbin chase in the present paper is $2.0 \mathrm{~mm}$ and is narrower than that in the previous $\operatorname{paper}^{[1]}(4.5 \mathrm{~mm})$. The same is true with the dimension of the hood entrance. Comparing both results, it is clear that the efficiency of the yarn suction in the present paper is better than that in previous paper ${ }^{[1]}$. This may be because, as shown later, the air flow pattern in the canal in the present experiment is more effective for yarn end retrieving by considering the pressure difference between the bobbin chase and the hood and the pressure gradient curves on both walls of the bobbin chase and the hood.

Fig. 2 shows that, in the cylindrical Hood D' at $1.6 \mathrm{~m}^{3} / \mathrm{min}$, the efficiency of yarn end retrieving is $75 \%$ when the broken ends are placed at the top of the bobbin chase, although it reaches $100 \%$ when the broken ends of the yarn are placed at the middle and at the bottom of the bobbin chase. For those Hood A', B' and C' which have conical angles, the retrieving efficiency is nearly $100 \%$ at $1.6 \mathrm{~m}^{3} / \mathrm{min}$ when the broken ends are placed at any where on the bobbin chase. There is the difference between with and without the conical angle. In Hood $A^{\prime}$, the retrieving efficiency is higher when the broken ends are placed at the top of the bobbin chase than that when placed at the middle or at the bottom and is $100 \%$ at $1.4 \mathrm{~m}^{3} / \mathrm{min}$. But the efficiency can't reach $100 \%$ when the broken ends are placed at the middle or at the bottom even at $Q=1.6 \mathrm{~m}^{3} / \mathrm{min}$. The retrieving efficiency for Hood $B^{\prime}$ is nearly constant independent of the the place of the broken end if $Q$ is more than $1.0 \mathrm{~m}^{3} / \mathrm{min}$. Even the flow rate is $1.6 \mathrm{~m}^{3} / \mathrm{min}$, the end retrieving is not sure. Though the retrieving efficiency for Hood C' depends largely on the place of the broken end, it increases greatly as the flow rate increases when the broken ends are placed at the top of the bobbin chase. The yarn end retrieving is sure at $1.6 \mathrm{~m}^{3} / \mathrm{min}$ even when the broken ends are placed at any where.

The above shows that the yarn end retrieving using Hood $C^{\prime}$ has the highest reliability when the air flow rate increases to $1.6 \mathrm{~m}^{3} / \mathrm{min}$. 


\subsection{Peculiarity of each hood type}

The pressure distributions on the inner surface of the hood and the bobbin using the model bobbin are measured and shown in Fig. 3 when the gap clearance $o$ is $2.0 \mathrm{~mm}$. The details are as follows:

a) $\operatorname{Hood} \mathrm{A}^{\prime}$

In this case, as the gap clearance $o$ is constant along the whole canal, the cross sectional area of the canal decreases inversely proportional to the position when it goes up from the entrance to the top of the chase along the bobbin axis, while the wall pressure distribution curve is parabolic having the vertex at the bottom of the chase. Therefore, from the continuity equation, the mean velocity in the cross section increases proportionally to the distance from the bottom of the chase. The difference between the pressure on the hood inner surface and that of the bobbin is comparatively little. In the canal, the flow does not appear such violent flow as a staggered flow or a rush flow against the wall. So, the flow pattern is thought to be a smooth acceleration flow.

The retrieving efficiency is good only at the top of the bobbin chase due to the effect of the hood skirt at the entrance. The wall pressure on the bobbin is lower than that on the hood in the range from the bottom to the middle of the chase, and is higher near the top of the chase. So it is considered that the hood skirt at the chase bottom causes the flow biased to the hood at the chase bottom and adversely at the middle of the chase. Both the flow biased to the bobbin and the accelerated flow are created at the chase top, and they induce the yarn sloughing form the bobbin. Such phenomenon often appears in the suction experiment.

b) Hood B'

The position of the upper corner of this conical hood is different from Hood $A^{\prime}$, and is a little higher than the top of the bobbin chase as shown in Fig. 3 (b). The pressure variation is larger than Hood $A^{\prime}$ along the whole chase, and the crossing of the pressure gradient curves and deep pressure drops are seen at the middle of the chase. Therefore, at this position a sudden change of the flow pattern is thought to exist. In the canal from the upper corner of the conical food to the top of the bobbin chase, the pressure distribution curves on both walls rapidly rise as the cross sectional area increases, and the pressure on the hood wall is lower than the pressure on the bobbin wall. So, it seems that the canal flow from the entrance to the middle of the chase is a flow biased to the hood due to the effect of the hood skirt, from the middle to the top a rapid accelerated flow biased to the bobbin chase, and in the expanding canal at the chase top is a flow biased to the bobbin chase. By such a flow pattern as staggered flow, the retrieving efficiency is constant along the whole bobbin chase and is comparatively good as shown in Fig. 2 (b). Even the flow resistance of Hood B' is lower than that of Hood A'. Consequently, Hood B' is considered to be

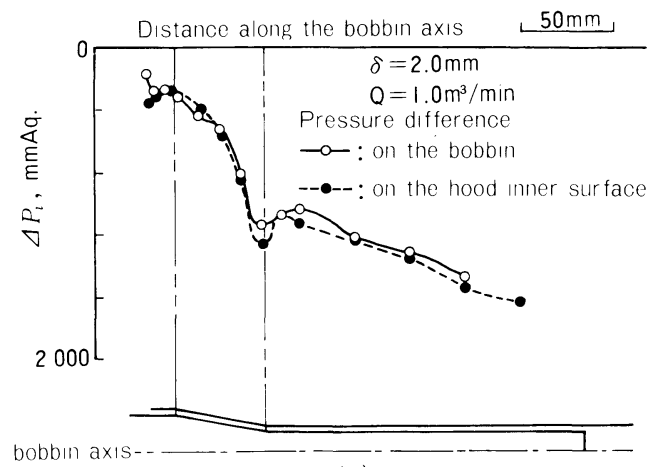

(a)

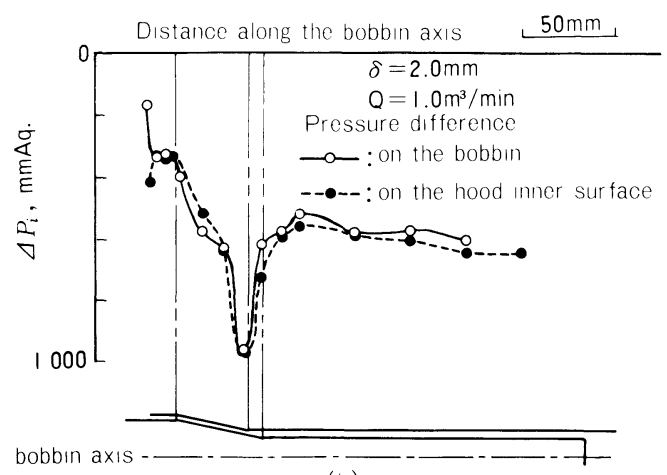

(b)
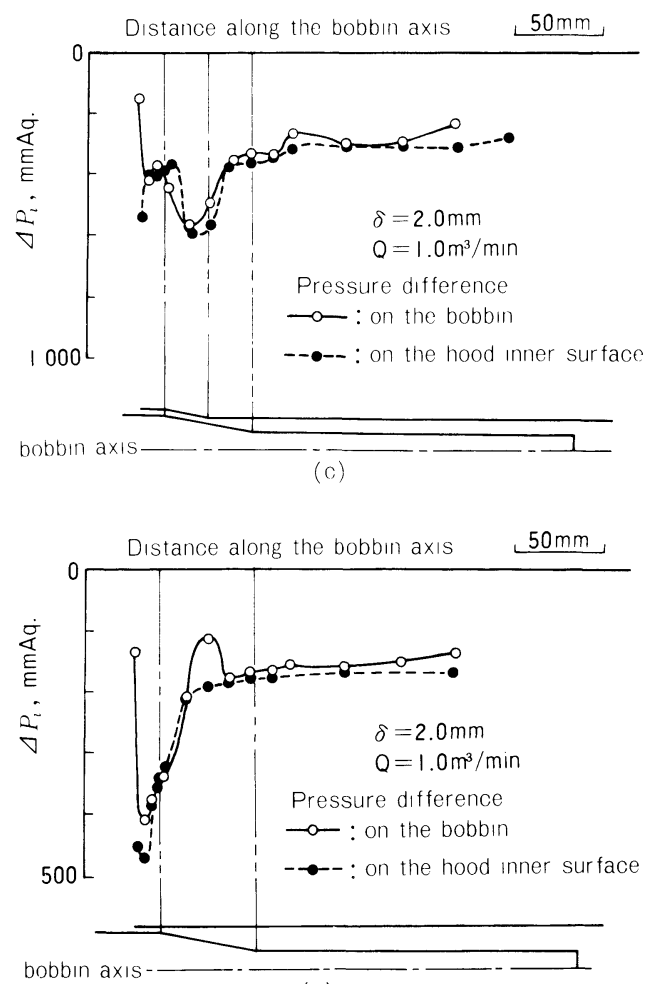

(d)

Fig. 3 Wall pressures along the bobbin axis (a) Hood A' (b) Hood B' (c) Hood C' (d) Hood D' 
an effective type for yarn retrieving.

c) Hood C'

Hood C' is similar to Hood B', but its top corner is at the middle of the chase as shown in Fig. 3 (c), which shows that the pressure on the bobbin wall drops and the pressure on the hood wall rises at the bottom of the bobbin chase, that both pressure distribution curves drop in the range from the bottom to the middle of the bobbin chase and cross there, that both pressures become the lowest at the middle chase but rise simultaneously thereafter. The wall pressure distributions change largely in the whole canal along the bobbin chase. So, it is guessed that the flow at the bottom rushes to the hood and the staggered flow appears towards the middle and that from the middle to the top of the bobbin chase, the flow rushes a little biased to the hood. These flow patterns at the bottom and at the middle of the chase are effective to yarn retrieving. The flow pattern at the chase top may also be effective for yarn retrieving if the flow disturbance is induced with acceleration. The comparison of Hood A' with Hood B' shows that, as the flow resistance of Hood $C^{\prime}$ is lower, Hood $C^{\prime}$ is thought to be the best type for yarn end retrieving.

\section{d) Hood D'}

Hood D' shapes much different from Hoods A', B' and $C^{\prime}$ as it has no parallel part. Its cross sectional area increases from the entrance to the top of the bobbin chase. As observed in Fig. 3 (d), the wall pressure distribution curves rapidly increase in the range from the bottom to the top of the bobbin chase, and the wall pressure of the bobbin is higher than that of the hood along from the middle to the top of the bobbin chase. This fact suggests that a speed reducing flow biased to the hood wall is created there, and that the effective yarn end retrieving can not be expected. Only at the bottom of the bobbin chase, the flow is disturbed by rapid expansion from the narrow canal, and is partially effective for yarn end retrieving. This agrees well with the suction performance shown in Fig. 2 (d).

\subsection{Effect of flow rate upon yarn end retrieving}

The flow rate effect upon the retrieving efficiency is remarkable in Hood $C^{\prime}$ as shown in Fig. 2 (a)-(d), and the suction efficiency is $100 \%$ when the flow rate is $1.6 \mathrm{~m}^{3} / \mathrm{min}$. Therefore, the effect of the flow rate upon the flow pattern and the yarn end retrieving will be considered with Hood C'.

The wall pressure distributions shown in Fig. 4 are the case in which the gap clearance $\delta$ is $2.0 \mathrm{~mm}$ and the flow rate is from 1.2 to $1.6 \mathrm{~m}^{3} / \mathrm{min}$. The case of $Q=1.0 \mathrm{~m}^{3} / \mathrm{min}$ is shown in Fig. 3 (c). Comparing with Fig. 3 (c) and Fig. 4, it is shown that the peculiarity of Hood C' becomes more remarkable over the whole resion as the flow rate increases. Therefore, the yarn end retrieving is superior by increasing flow rate. However, as the excessive flow brings about much energy loss, the selection of the optimum flow rate is needed as described in the previous paper ${ }^{[2]}$.
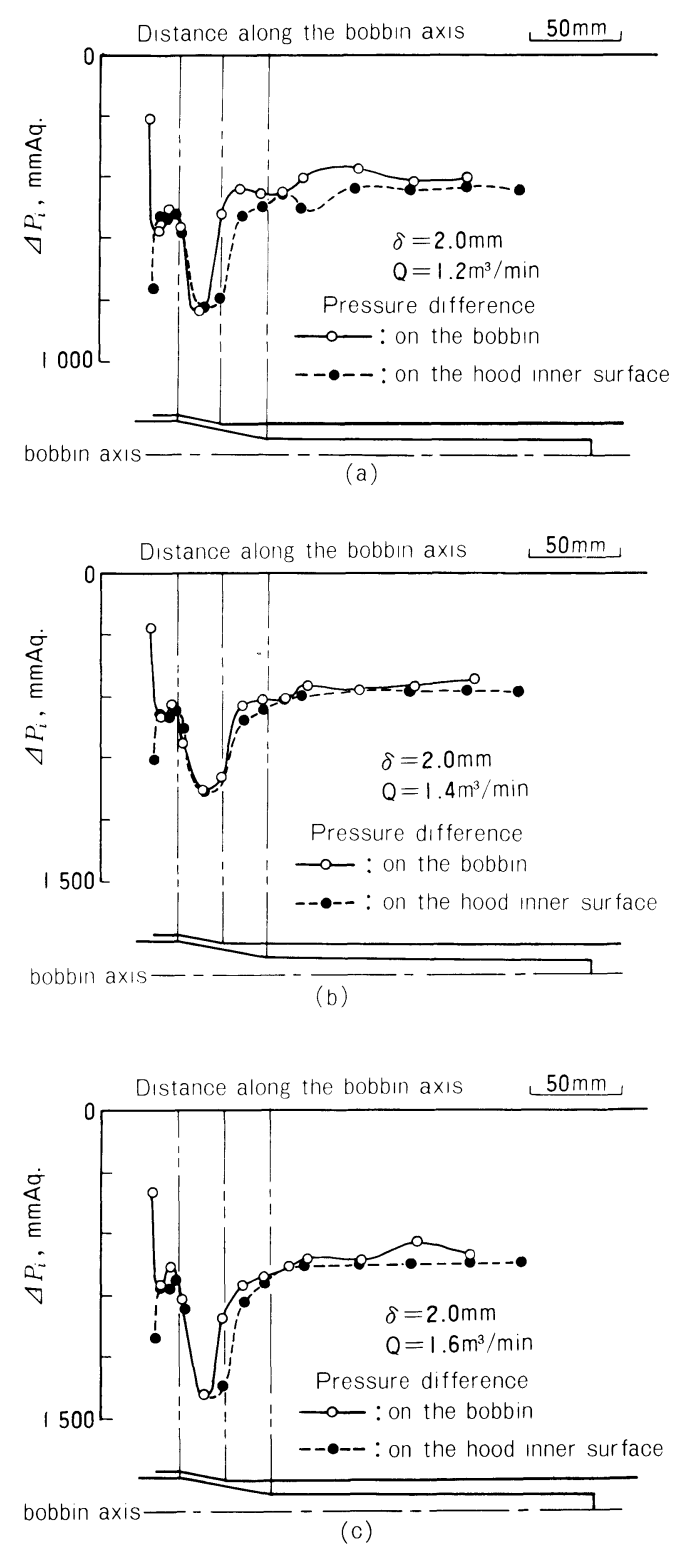

Fig. 4 Wall pressures along the bobbin axis (Hood C')

3.3 Effect of gap clearance upon yarn end retrieving

The wall pressure distributions are shown in Figs. 5, 6, 7 and 8 when $Q=0.7 \mathrm{~m}^{3} / \mathrm{min}$, using Hoods A', B' and C'. The canal shape itself can be changed by changing the gap clearance. Fig. 8 was obtained in the previous paper ${ }^{[1]}$ in case of $Q=0.7 \mathrm{~m}^{3} / \mathrm{min}$ and the gap clearance $=1.0 \mathrm{~mm}$. The peculiarity is the same in both shown in Fig. 5 (a) and Fig. 8, that is to say, they have both the reduction of the cross sectional area in the canal at the chase bottom and the expansion at the chase top.

The common gap clearance is $2.0 \mathrm{~mm}$ in the present experiments as shown in Fig. 5(b), Fig. 6 (b) and Fig. 7 (b), and 

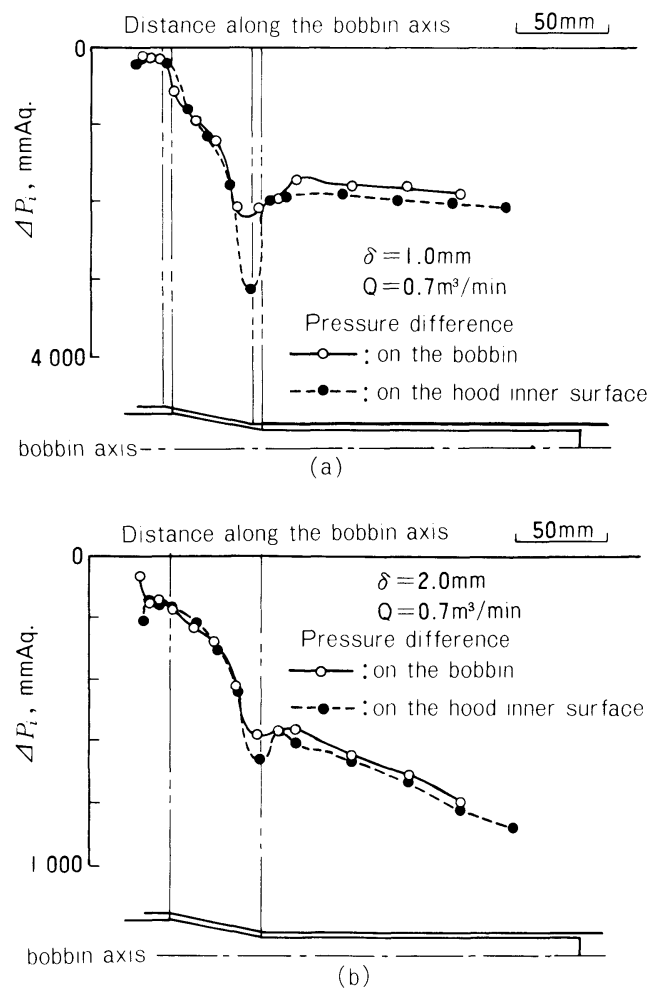

Fig. 5 Wall pressures along the bobbin axis (Hood $\left.A^{\prime}\right)$
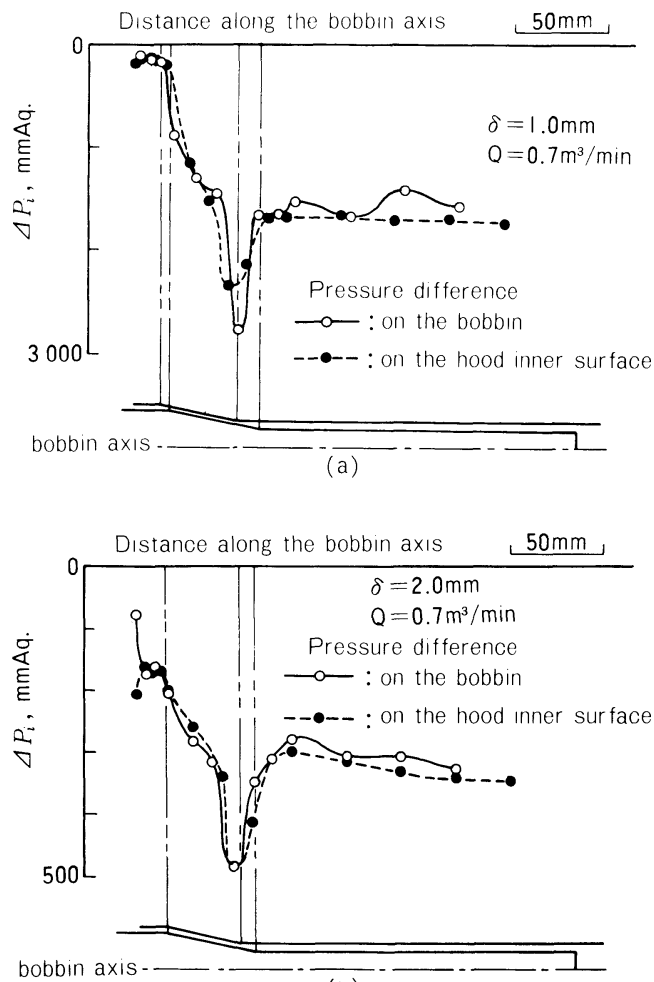

(b)

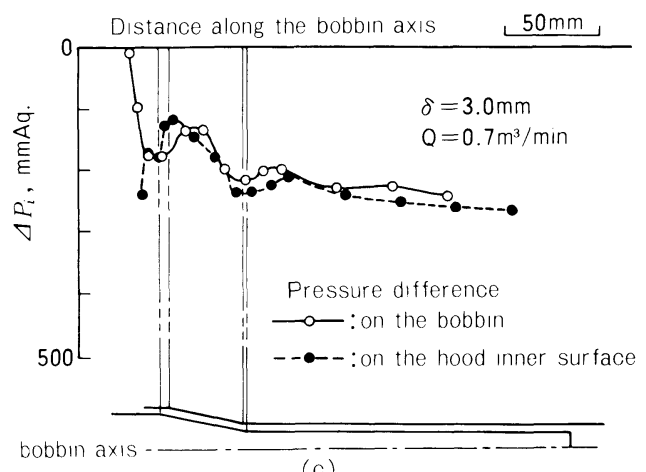

(c)

Fig. 6 Wall pressures along the bobbin axis (Hood B')
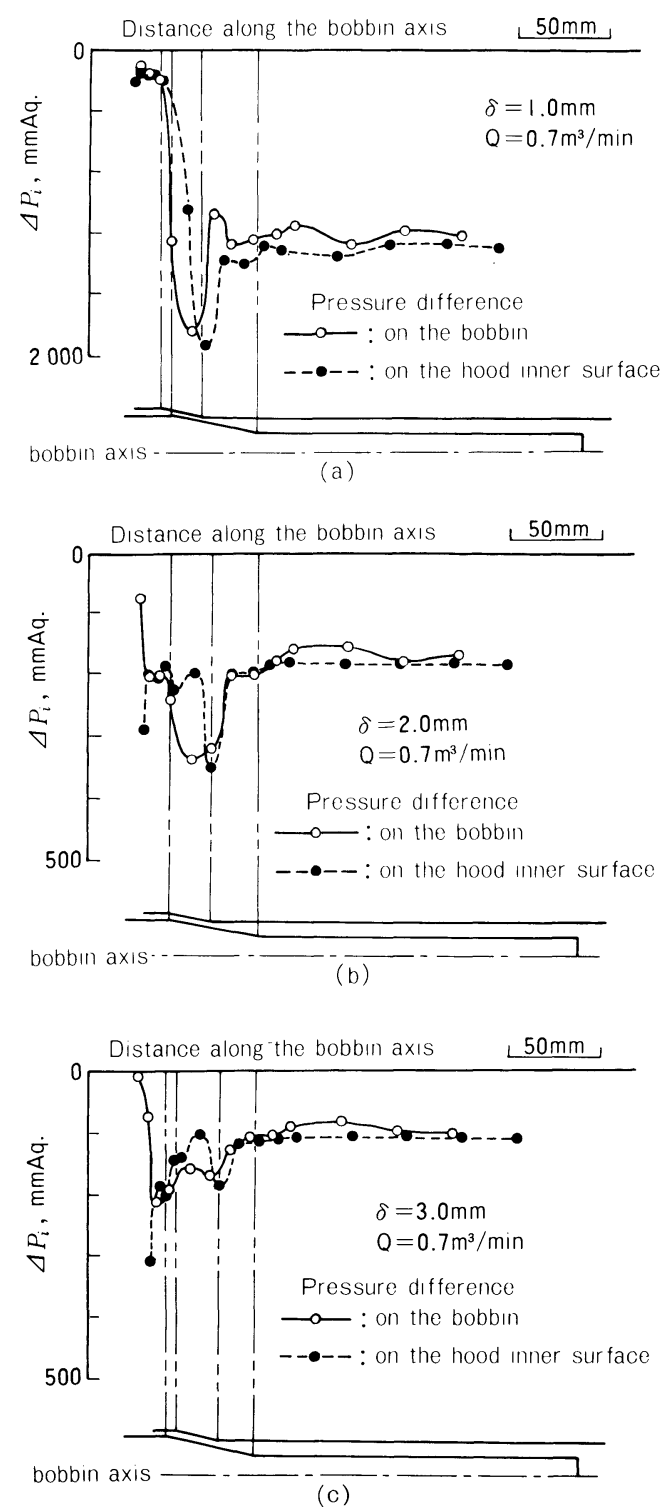

Fig. 7 Wall pressures along the bobbin axis (Hood C') 
the consideration in these cases was already described. The pressure drop is extremely deep when the gap clearance is narrow as shown in Fig. 5 (a), Fig. 6 (a) and Fig. 7 (a). By enough acceleration from the deep pressure drop, the suction performance becomes better, but the yarn sloughing appears and energy loss increases. When the gap clearance is wide as shown in Fig. 6 (c) and Fig. 7 (c), the pressure drop is not enough for yarn suction. These cases, same as Hood D', are not effective for yarn end retrieving.

Consequently, it is considered that an optimum gap clearance with an effective hood is needed to attain effective and economical yarn end retrieving.

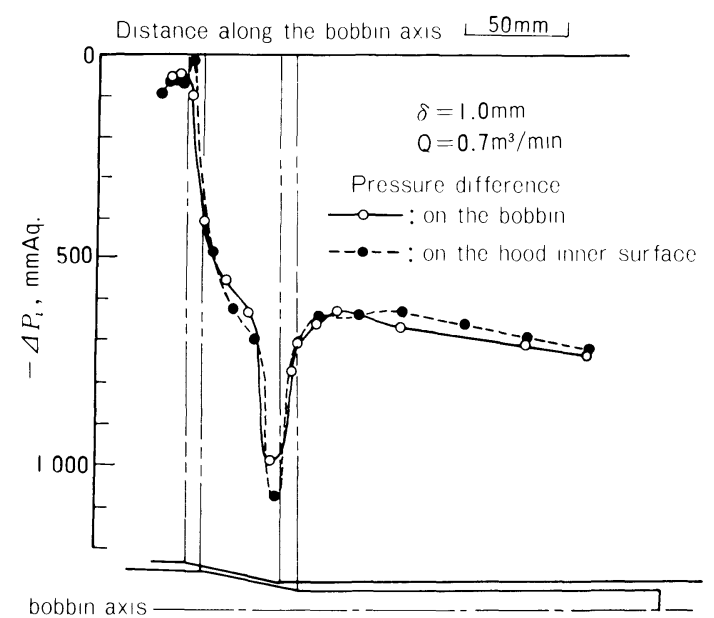

Fig. 8 Wall pressures along the bobbin axis (in previous paper $[1]$ )

\section{Conclusion}

Studies for yarn end retrieving by air flow using bobbins with suitable hoods were carried out, and the effective canal and the flow pattern were reported ${ }^{[1,2]}$. They pointed out that the effect of the canal shapes at the top of the bobbin chase upon the retrieving efficiency should be considered.

In this paper the retrieving efficiency using actual bobbins was observed with various hoods and the peculiarity of the flow pattern in these canals was found. Followings are the main results obtained:

(1) In case of Hood A', the air flow is monotonously accelerated along the whole canal, and the yarn sloughing may appear. The flow resistance is large.

(2) In case of Hood B', the retrieving efficiency becomes better at any location of the yarn end as the flow rate increases. The flow resistance is weaker than that of Hood A. But the retrieving efficiency could not be made $100 \%$.

(3) In case of Hood C', the retrieving efficiency was not better at the top of the bobbin chase when the flow rate was low. But the efficiency rapidly rised up to $100 \%$ as the flow rate increased. As the flow resistance was weaker than that of Hood B', Hood C' seems to be the most effective upon yarn end retrieving.

(4) In case of the cylindrical Hood D', as the canal monotonously expands, the flow speed rapidly reduced and the flow pattern was not effective for yarn end retrieving.

\section{References}

[1] T. Hirai, T. Katayama, T. Tanaka, N. Heya and S. Fukai; J.Text. Mach.Soc. Japan, 26, 4 (1980) 115.

[2] T. Hirai, T. Katayama, T. Tanaka, N. Heya and S. Fukai; J. Text. Mach. Soc. Japan, 27, 1 (1981) 22. 